inflammation research, Göteborg, Sweden; ${ }^{3}$ Karolinska Institute, Department of Biosciences and Nutrition, Solna, Sweden; ${ }^{4}$ the Sahlgrenska Academy at University of Gothenburg, Department of Chemistry and Molecular Biology, Gothenburg, Sweden

Background: The oncogenic protein survivin is a marker of severe rheumatoid arthritis (RA). High serum levels of Survivin predict progressive joint damage ${ }^{1}$ and poor treatment response ${ }^{2}$.

Objectives: To study the role of survivin in the transcriptional regulation of phenotype in $\mathrm{CD}^{+} \mathrm{T}$ cells.

Methods: $\mathrm{CD}^{+} \mathrm{T}$ cells of RA female patients were isolated from the perpheral blood. Activated $\mathrm{CD} 4^{+}$cells were treated with survivin inhibitor YM155. Transcriptional analysis was done by RNAseq (Illumina) and conventional qPCR. Chromatin of CD4 cells was immunoprecipitated using polyclonal antibodies to survivin and subjected to deep sequencing (survivin ChIPseq, Hiseq2000, Illumina) and aligned to GRCh38. Statistical analysis of differentially expressed genes (DEG) was done in R-studio using Benjamini-Hochberg adjustment for multiple testing (Bioconductor, DESeq2 package).

Results: Survivin ChIPseq of the activated $\mathrm{CD}^{+} \mathrm{T}$ cells was enriched with the genes engaged in regulatory transcription factor specific DNA binding (GO:0000987, adj $\mathrm{p}=0.0005$ ) and RNA polymerase II regulatory transcription (GO:0000978, adj $p=0.0004)$. Among survivin targets were the genes of HOX-B cluster and TALE family proteins MEIS, PKNOX and PBX1 controlling early leukopoesis and T cell maturation. Inhibition of survivin in PBMC resulted in significant upregulation of PBX1 $(p=0.023)$, MEIS3 $(p=0.0036)$, similar tendency was observed for HOXB6 and HOXC4 genes. RNAseq analysis CD4 cells of RA patients with different transcription of PBX1, identified 1636 genes (adj $\mathrm{p}<10-5)$. BIRC5, coding for survivin, was 8.3 folds higher in $\mathrm{CD} 4^{+}$cells with low PBX1 $(p=0.0005)$. Among the core transcription factors of $T$ helper cell differentiation, we identifed NF-kB1 and NF-kB2, TBX21, IRF4, IRF8 and STAT3, BATF and BATF3. This followed by significantly higher TNF, IFNg and IL17A and IL17F in PBX1lo CD4 T cells. The pathway enrichment analysis of DEG identified strong over-representation of cytokine-specific genes (GO:005125, GO:0005126, GO:0048018, GO:0030545, FDR q-values $\left.10^{-12}-10^{-9}\right)$. The genes of IL4, IL5, IL13, IL9, IL3 and CSF2 located within the chromosome 5 were common for all GO-lists, and were higher in PBX1lo, but none of those genes was identified by survivin-ChIPseq or PBX1-ChIPseq. Analysis of ChIPseq data identified the genes of STAT3, IRF4, IRF8 and BATF as common targets for PBX1 and survivin.

Conclusion: This genome-wide analysis indicates that survivin regulates transcription of the TALE family protein PBX1 in CD4+ T cells, which has essential effect for differentiation and phenotype of Th subsets. Low PBX1 in RA patients is associated with terminally differentiated effector CD4+ T cells.

References:

[1] Svensson, B. et.al. Smoking in combination with antibodies to cyclic citrullinated peptides is associated with persistently high levels of survivin in early rheumatoid arthritis: a prospective cohort study. Arthritis Res Ther 16, R12 (2014).

[2] Levitsky, A. et.al. Serum survivin predicts responses to treatment in active rheumatoid arthritis: a post hoc analysis from the SWEFOT trial. BMC Med 13, 247 (2015)

Disclosure of Interests: None declared

DOI: 10.1136/annrheumdis-2020-eular.5426

\section{THU0038 \\ DIFFERENTIAL ROLES OF TNFRI AND TNFRII IN THE MORPHOLOGY OF SECONDARY LYMPHOID ORGANS}

K. Jeucken $^{1,2}$, J. P. Van Hamburg ${ }^{1,2}$, S. Tas ${ }^{1,2} .{ }^{1}$ Amsterdam UMC, Experimental Immunology - Clinical Rheumatology and Immunology, Amsterdam, Netherlands; ${ }^{2}$ Amsterdam Infection and Immunity Institute, Amsterdam, Netherlands

Background: Tumour necrosis factor (TNF) induced signaling events are important in lymphoid organ development and function, both in health and in immune-mediated inflammatory diseases such as arthritis. Important receptors involved in this process include TNF receptors (R) I and II that exert distinct functions. Mice overexpressing transmembrane (tm)TNF develop various features of chronic inflammation, including arthritis, that are mediated via TNFRI and/or TNFRII.

Objectives: To investigate the importance of TNFRI and TNFRII in spleen and peripheral lymph node (PLN) morphology in mice overexpressing tmTNF. Methods: Spleen and PLN were collected from 13 week old mice with the following genotypes: WT, tmTNF transgenic heterozygotes (tmTNF tg HET), WT $\times$ TNFRI $^{-/}$, tmTNF tg HET $\times$TNFRI $^{-1}$, WT $\times$ TNFRII $^{-1-}$ and tmTNF tg HET $x$ TNFRII ${ }^{-1-}$. Spleens were cut and stained by immunofluorescence for $B$ and T cell markers, imaged by confocal microscopy and analyzed using FIJl software. PLN were used for whole mount tissue staining for B and T cell markers, cleared, 3D imaged by light sheet microscopy and analyzed using Imaris software.

Results: Although no macroscopic differences were observed, spleens of tmTNF tg HET animals exhibited an altered morphology characterized by smaller follicles $\left(69,73 \pm 72,25 \mu \mathrm{m}^{2}\right)$ compared to spleens of WT mice $(139,3 \pm 71,36$ $\mu \mathrm{m}^{2}$ ), which was accompanied by a decrease in central T cell areas as measured by $\%$ of the total follicle $(15,22 \pm 9,685$; WT $26,60 \pm 10,46)$. WT mice lacking either TNFRI or TNFRII had a morphology comparable to WT (follicle size: TNFRI $234,0 \pm 166,9 \mu^{2}$; TNFRII $154,2 \pm 87,92 \mu \mathrm{m}^{2} ; \%$ of T cell area: TNFRI $27,81 \pm$ 11,03; TNFRII 28,14 $\pm 19,66)$. tmTNF $\operatorname{tg} \times$ TNFRI $^{-/-}$exhibited a normal spleen architecture (follicle size: $239,2 \pm 250,5 \mu \mathrm{m}^{2}$; \% of T cell area: $29,92 \pm 11,46$ ), whereas the spleen follicles of tmTNF $\operatorname{tg} \times$ TNFRII $^{-/}$mice had $\mathrm{T}$ cell areas $(17,31$ $\pm 8,88)$ that were comparable to tmTNF tg HET mice.

The size of PLN of tmTNF $\mathrm{tg}$ HET mice $\left(3,74 \pm 1,31 \mathrm{~mm}^{3}\right)$ was increased compared to WT $\left(2,41 \pm 0,60 \mathrm{~mm}^{3}\right)$, also in combination with deficiency of TNFRI $\left(3,98 \pm 2,07 \mathrm{~mm}^{3}\right)$ or TNFRII $\left(4,01 \mathrm{~mm}^{3}\right)$. tmTNF tg HET mice had an increase in absolute $B$ cell volume $\left(0,86 \pm 0,33 \mathrm{~mm}^{3}\right)$ vs WT $\left(0,51 \pm 0,07 \mathrm{~mm}^{3}\right)$ but no change in B cell area as \% of total PLN volume. The increased B cell volume was critically dependent on TNF-RII (tmTNF tg HET x TNFRII ${ }^{-/-}(14,87)$. Interestingly, whereas $B$ cell area morphology of tmTNF tg HET, tmTNF tg HET $x$ TNFRII $^{-1-}$ and WT $x$ TNFRII ${ }^{-1-}$ PLN was similar to WT, TNFRI deficiency caused profound alterations in the morphology of the $\mathrm{B}$ cell areas that appeared as one peripheral layer of $\mathrm{B}$ cells covering a central $\mathrm{T}$ cell area rather than properly developed follicles.

Conclusion: The current study demonstrates that overexpression of tmTNF leads to an aberrant spleen architecture, characterized by smaller follicles and a decrease in central T cell areas, which is critically dependent on TNFRI. In addition, overexpression of tmTNF results in enlarged PLN and increased total B cell volume, which is dependent on TNFRII, whereas TNFRI is more important in the proper organization of $\mathrm{B}$ cell follicles. Overall, this study employing different stateof-the-art (3D) imaging techniques highlights the importance of tmTNF-TNFR-induced signaling events in secondary lymphoid organ morphology and function and reveals distinct roles for TNFRI and II. Advancing our knowledge in this field might provide a better understanding of the pathophysiology of TNF-associated diseases such as arthritis, which may be important to develop new or improved treatment strategies.

Disclosure of Interests: None declared

DOI: 10.1136/annrheumdis-2020-eular.5873

\section{\begin{tabular}{|l|l}
\hline THU0039 DECIPHERING DISEASE-RELEVANT T CELL \\
\hline
\end{tabular} SUBSETS IN RHEUMATOID ARTHRITIS IDENTIFIES A NOVEL CELLULAR SUBSET OF PATHOGENETIC IMPORTANCE IN THERAPEUTIC RESISTANCE.}

M. Nyirenda ${ }^{1}$, I. Mcinnes ${ }^{1}$, C. Goodyear ${ }^{1} .{ }^{1}$ Institute of Infection, Immunity and Inflammation, College of Medical, Veterinary and Life Sciences, Glasgow, United Kingdom

Background: Aberrant $\mathrm{T}$ cell responses are key in driving autoimmunity and are commonly associated with rheumatoid arthritis (RA). Unravelling pathways of importance in therapeutic partial response and failure is of critical importance, as this will potentially provide new insights into key drivers of immune-mediated pathogenesis.

Objectives: To delineate disease-relevant $T$ cell subsets in RA and assess their potential to act as cellular markers amenable to precision medicine approaches, particularly in the context of therapeutic partial or non-response.

Methods: FACS-based immunophenotyping and ex-vivo functional response profiles of $\mathrm{CD} 4^{+} \mathrm{CD} 161^{+} \mathrm{CCR} 2^{+} \mathrm{CCR} 5^{+} \mathrm{T}$ cells were performed in peripheral blood mononuclear cells (PBMC) obtained from patients with RA and healthy controls, using previously characterised methodologies. RA patients fulfilled the 2010 ACR/EULAR criteria for RA. All samples were obtained after written consent, with the appropriate ethical approvals in place.

Results: RA patients harboured a higher frequency of $\mathrm{CCR} 2^{+} \mathrm{CCR} 5^{+}$cells within the $\mathrm{CD} 4^{+} \mathrm{CD} 161^{+} \mathrm{T}$ cell compartment compared with healthy controls. In RA patients this $T$ cell subset had a higher proportion of cells that secrete pro-inflammatory cytokines such as IL-17A, GM-CSF, IFN- $\gamma$, and TNF. Importantly, the $\mathrm{CD} 4^{+} \mathrm{CD} 161^{+} \mathrm{CCR} 2^{+} \mathrm{CCR} 5^{+} \mathrm{T}$ cell subset was significantly increased in DMARD non-responders compared to both responders and healthy controls. Moreover, in DMARD non-responders, these cells had a propensity to express increased proportions of pro-inflammatory cytokines. Notably, there was also a significant increase in the ratio of effector: regulatory $\mathrm{T}$ cell (Teff: Treg) compared to both responders and healthy controls. In addition, the $\mathrm{CD} 4^{+} \mathrm{CD} 161^{+} \mathrm{CCR} 2^{+} \mathrm{CCR} 5^{+}$ $T$ cell subset was less responsive to suppression by Tregs. In further support of a role for this $T$ cell population in disease pathogenesis, the frequency of $\mathrm{CD} 4^{+} \mathrm{CD} 161^{+} \mathrm{CCR} 2^{+} \mathrm{CCR} 5^{+} \mathrm{T}$ cells significantly correlated with disease activity, as measured by the DAS28 $\left(R^{2}=0.65 ; p=0.003 ; n=11\right)$. 
Conclusion: Combined, our findings suggest that the $\mathrm{CD} 4^{+} \mathrm{CD} 161^{+} \mathrm{C}$ $\mathrm{CR}^{+}{ }^{+} \mathrm{CR} 5^{+} \mathrm{T}$ cell subset represents a substantially abnormal $\mathrm{T}$ cell subset in $R A$, exhibiting exaggerated pro-inflammatory responses, numerical abundance relative to Tregs, and resistant to regulation by Tregs. The $\mathrm{CD} 4^{+} \mathrm{CD} 161^{+} \mathrm{C}$ $\mathrm{CR} 2^{+} \mathrm{CCR} 5^{+} \mathrm{T}$ cell subset appears to be a marker of therapeutic response status in RA, via its contribution to disease pathology and highlights this subset as a potential therapeutic target in RA.

References:

[1] Mclnnes IB, Schett G. The pathogenesis of rheumatoid arthritis. $N$ Engl J Med. 2011;365(23):2205-19.

[2] Mexhitaj I, Nyirenda MH, Li R, O’Mahony J, Rezk A, Rozenberg A, et al. Abnormal effector and regulatory $T$ cell subsets in paediatric-onset multiple sclerosis. Brain. 2019;142(3):617-32.

[3] Cosmi L, Cimaz R, Maggi L, Santarlasci V, Capone M, Borriello F, et al. Evidence of the transient nature of the Th17 phenotype of $\mathrm{CD} 4^{+} \mathrm{CD} 161^{+} \mathrm{T}$ cells in the synovial fluid of patients with juvenile idiopathic arthritis. Arthritis Rheum. 2011;63(8):2504-15.

Disclosure of Interests: Mukanthu Nyirenda: None declared, lain Mclnnes Grant/research support from: Bristol-Myers Squibb, Celgene, Eli Lilly and Company, Janssen, and UCB, Consultant of: AbbVie, Bristol-Myers Squibb, Celgene, Eli Lilly and Company, Gilead, Janssen, Novartis, Pfizer, and UCB, Carl Goodyear: None declared

DOI: 10.1136/annrheumdis-2020-eular.5525

\section{THU0040 $\quad$ PROTEINASE 3-REACTIVE B CELL RECONSTITUTION AFTER TREATMENT WITH RITUXIMAB FOR ANCA- ASSOCIATED VASCULITIS}

A. Berti ${ }^{1}$, S. Hillion ${ }^{2}$, A. Hummel ${ }^{3}$, E. Carmona ${ }^{3}$, T. Peikert ${ }^{3}$, C. Langford ${ }^{4}$, P. A. Merkel ${ }^{5}$, P. Monach ${ }^{6}$, P. Seo ${ }^{7}$, R. Spiera ${ }^{8}$, E. W. St. Clair ${ }^{9}$, F. Fervenza $^{3}$, K. Harris ${ }^{10}$, J. H. Stone ${ }^{11}$, J. O. Pers ${ }^{2}$, U. Specks ${ }^{3}$, D. Cornec ${ }^{2}$ on behalf of the RAVE group. ${ }^{1}$ Santa Chiara Hospital, Trento, Italy; ${ }^{2}$ Université de Bretagne Occidentale, Brest, France; ${ }^{3}$ Mayo Clinic, ROCHESTER, United States of America; ${ }^{4}$ Cleveland Clinic, Cleveland, United States of America; ${ }^{5}$ University of Pennsylvania, Philadelphia, United States of America; ${ }^{6}$ Brigham and Women's Hospital, Boston, United States of America; ${ }^{7}$ Johns Hopkins Medicine, Baltimore, United States of America; ${ }^{8}$ Hospital for Special Surgery, New York, New York, United States of America; ${ }^{9}$ Duke University, Durham, United States of America; ${ }^{10}$ immune Tolerance Group, Bethesda, United States of America; ${ }^{11}$ Massachusetts General Hospital, Boston, United States of America

Background: Proteinase 3 (PR3)-reactive B cells are present in PR3-ANCA-associated vasculitis (AAV) at levels higher than healthy controls.

Objectives: To evaluate the dynamics of the PR3-reactive B cell repopulation in patients with PR3-AAV after treatment with rituximab, and to analyze possible associations between these immunological changes and long-lasting remissions.

Methods: We analyzed all available frozen peripheral blood mononuclear cells $(n=148$ ) from 23 randomly-selected PR3-AAV patients who participated in the RAVE trial and achieved complete remission (BVAS $=0$, prednisone $=0$ ) after treatment with rituximab.

We measured PR3-reactive $B$ cells and the relative subsets by a multi-color flow cytometry panel including CD19, IgD, CD27, CD38, CD24, and a biotinylated PR3 revealed by fluorescent streptavidin. The clinical data of the trial were correlated with flow-cytometry data.

Results: 10/23 (43\%) patients relapsed during the follow up, 8/10 relapses were severe. At baseline, clinical features, PR3-ANCA levels, \% of total PR3-reactive $B$ cells and PR3-reactive B cell subsets were similar between relapsers and non-relapsers. All patients were followed until the end of the trial, for a mean of 44 months (25-75\%IQR 31-54), without difference in follow-up time between relapsers and non-relapsers $(p=0.98)$.

The majority of patients had $B$ cell repopulation at 12 (range 12-24) months after rituximab. At the time of $\mathrm{B}$ cell repopulation, transitional (CD19+CD24+CD38+) and naïve (CD19+CD27+lgD-) B cells were higher compared to baseline, while total plasmablasts (PB) were unchanged, and mature B cells significantly decreased in both relapsers and non relapsers. PR3-reactive $B$ cells reappeared in all the patients, and the \% of PR3-reactive of B cells were higher at the B cell repopulation visit compared to baseline $(5.82 \%$ vs $4.25 \%, p<0.05)$, while total $B$ cells were lower $(66 / \mu \mathrm{L}$ vs $151 / \mu \mathrm{L}, \mathrm{p}<0.01)$, regardless of future relapse.

Within PR3-reactive B cells, only the \% of PB (CD19+CD27+CD38+PR3+) were higher in relapsers vs. non-relapsers (median [25-75\%IQR]; 1.95\% [1.315-3.845] vs $0.84 \%$ [0.05-1.66], $\mathrm{p}=0.022$ ) and severe relapsers vs non-severe relapsers (2.165\% [1.66-4.315] vs $0.84 \%$ [0.1-1.74], $p=0.015)$. Time-to-relapse and time-to severe-relapse were significantly shorter in patients with circulating PR3-PB higher than the median value of the cohort $(1.6 \%)$ during $B$ cell reconstitution (Figure 1A-B).
Conclusion: In PR3-AAV, during $B$ cell reconstitution after rituximab, the total fraction of PR3-B cells increases, due to the expansion of the transitional and naïve B cell compartments. Circulating PR3-PB within PR3-B cells are enriched in the peripheral blood of relapsing and severely relapsing patients compared to non-relapsing patients. Higher levels of PR3-PB after rituximab during B cell reappearance significantly increased the risk of subsequent relapse and severe relapse.

References:

[1] Cornec D, Berti A, Hummel A, et al. J Autoimmun. 2017
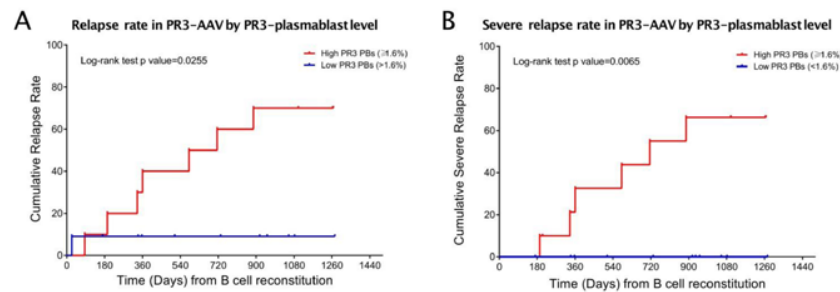

Disclosure of Interests: Alvise Berti: None declared, Sophie Hillion: None declared, Amber Hummel: None declared, Eva Carmona: None declared, Tobias Peikert: None declared, Carol Langford: None declared, Peter A. Merkel: None declared, Paul Monach: None declared, Philip Seo: None declared, Robert Spiera Grant/research support from: Roche-Genetech, GSK, Boehringer Ingelheim, Chemocentryx, Corbus, Forbius, Sanofi, Inflarx, Consultant of: Roche-Genetech, GSK, CSL Behring, Sanofi, Janssen, Chemocentryx, Forbius, Mistubishi Tanabe, E. William St. Clair: None declared, Fernando Fervenza: None declared, Kristina Harris: None declared John H. Stone Grant/research support from: Roche, Consultant of: Roche, Jacques-Olivier Pers: None declared, Ulrich Specks: None declared, Divi Cornec: None declared

DOI: 10.1136/annrheumdis-2020-eular.5169

\section{THU0041 IFNA AND IL21 PROMOTE DISTINCT POPULATIONS OF EFFECTOR B CELLS}

M. Boudigou ${ }^{1}$, A. Grasseau ${ }^{1}$, N. Chriti ${ }^{1}$, J. O. Pers ${ }^{1}$, L. Le Pottier ${ }^{1}$, S. Hillion ${ }^{1}$ ${ }^{1}$ Univ Brest, Inserm, LBAI, UMR1227, BREST, France

Background: B cells play a crucial role in the pathogenesis of systemic autoimmunity through various effector functions, including auto-antibody production secretion of pro-inflammatory cytokines and antigen presentation to $T$ cells. Interferon alpha (IFNa), mainly produced by innate cells (Menon et al., 2016) and interleukin (IL)-21 which is secreted by follicular helper T cells (Berglund et al., 2013), promote the generation of auto-reactive IgG-secreting plasma cells. However, it is suggested that IFNa participate also to the generation of regulatory B cells.

Objectives: To further understand the disturbing microenvironmental signals leading to autoimmune diseases, we aim to define a coherent framework integrating the B-cell subsets having different ability to respond to microenvironmental signals, and the signalling pathways driving the differentiation and the functional fate of $B$ cells.

Methods: Naïve and several populations of memory B cells were isolated from peripheral blood of healthy donors and differentiated in vitro in the presence of IL21 or IFNa. The phenotype and the expression of transcription factors were analysed by flow cytometry and molecular identity of these cells was further determined by transcriptomic approaches. Functional analyses were performed to assess the effector functions of $B$ cells and their potential regulatory effects on T cells.

Results: IFNa, in synergy with $\mathrm{CpG}$, promote the generation of CD27 $7^{\text {high }}$ CD38 ${ }^{\text {high }}$ plasmablasts (PB), mainly arising from memory subsets, but not IL21. However, IFNa and IL21 induce the transcriptional program of B-cell differentiation by up-regulating IRF4 and Blimp1 expression. Unlike IFNa, IL21 drive the

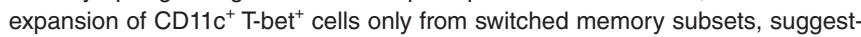
ing T-bet may be a footprint of long-lived memory B cells.

Even though subtle differences were observed in the antibody production between IFNa and IL21-stimulated memory B cells, naïve cells secreted less amount of IgM and IL10 when stimulated with IL21.

Transcriptomic studies are still in progress to further define the molecular profile of those distinct effector B cells.

Conclusion: Taken together, these findings suggest that IFNa promote a rapid differentiation of B cells into IL10 and Ig-secreting PB whereas IL21 contributes to the generation of T-bet ${ }^{+}$atypical pre-PB that may have a role in chronic autoimmune disorders. 